The impact of "consensus" as a new signpost in the process of decision making in multilateral negotiations is clearly highlighted ${ }^{\mathbf{2}}$. On the principle of "common heritage of mankind", Rembe, uses the African Land tenure systems of common ownership to illustrate African States' perception of UNCLOS III deliberations on the subject ${ }^{30}$.

The concluding chapter of this book evaluates African participation and contribution. It deals also with the new state of affairs that may arise when the Law of the Sea Conference comes to an end. Against this background, and to contain any doubts, African States are asked to be cautious in examining the legal implications of ratifying the Treaty, and the unforeseen consequences of implementing its provisions ${ }^{\mathbf{3 1}}$.

Rembe's book will remain a valuable reference work for students and teachers seeking to chart the course that the African States followed at UNCLOS III. It provides a conclusion that gives a guide in the quest for future work in the Law of the Sea, when all the hustle and bustle have calmed down.

Emmanuel G. Bello

Franz NusCheler/KLaus Ziemer

\title{
Politische Organisation und Repräsentation in Afrika
}

Walter de Gruyter, Berlin, New York, 1978, (Band II: Die Wahl der Parlamente und anderer Staatsorgane, hrsg. von Nohlen u. a., 2 Bände, 2507 S., 862 DM)

Dieser zweite Band - nach dem 1969 zu Europa erschienen - der Reihe „Die Wahl der Parlamente und anderer Staatsorgane“ beschränkt sich nicht auf diese enge Fragestellung, die die Autoren insbesondere für Afrika als ,,grotesk und museal“ (wohl mit Recht) bezeichnen (S. 1). Das vorliegende Werk will und muß mehr sein, will es den Charakter und das Funktionieren der politischen Systeme und des Willensbildungsprozesses in Afrika erfassen und auf den Begriff bringen.

Das Handbuch, das (begründet) Informationskompendium und Nachschlagewerk sein will (S. 3), besteht aus zwei Teilen: aus einer allgemeinen Einführung und aus Beiträgen zu 55 Ländern und Territorien. Die Verfasser zeichnen gemeinsam für die Einleitung und auch (individuell) für ca. die Hälfte der Länderbeiträge. Den Rest besorgten 16 weitere Autoren. In der umfangreichen ,Einleitung“ - die mit 200 Seiten eigentlich auch schon den Charakter einer eigenständigen Monographie hat - ziehen Nuscheler/Ziemer einen vergleichenden Querschnitt durch die politische Entwicklung des Kontinents und diskutieren dabei ausführlich und gekonnt - was in den Länderbeiträgen so nicht möglich wäre - die Theorien, Hypothesen und Erklärungsansätze der politischen Entwicklung. Sie gehen dabei zunächst auf die präkolonialen Gesellschafts-, Herrschafts- und Legitimitätsformen, auf die Praxis der Kolonisation und Dekolonisation sowie dazu den relevanten theoretischen Erklärungsversuchen, auf Genese und Organisation des afrikanischen Nationalismus, auf die Transformation der (kolonial hinterlassenen) Verfassungsmodelle (von der ,, Westminsterdemokratie" zum afrikanischen Präsidentialismus), schließlich auf Begründung, Entstehung und Entwicklung der Einparteisysteme und der Militärherrschaft als alternativer politischer Organisationsform ein. Den Abschluß dieses Teils bilden einige knappe Bemerkungen zum Stichwort ,,die afrikanischen Staaten als ,periphere Staaten““.

Mit dieser Einleitung ist Nuscheler/Ziemer ohne Zweifel eine der besten Einführungen in die politischen Systeme und die politische Entwicklung Afrikas gelungen, die durch die Hand

29 Ibid. p. 65.

30 Ibid. p. 53.

31 Ibid. p. 206. 
des Rezensenten gegangen ist. Sie ist vorzüglich geschrieben und damit gut lesbar. Unter Verarbeitung umfangreicher allgemeiner theoretischer und regionalspezifischer Literatur bietet sie sowohl für den Anfänger eine vorzügliche Einführung als auch für den Spezialisten eine anregende und bedenkenswerte Lektüre.

Dennoch sei auf zwei Defizite der Darstellung hingewiesen, die die obige Feststellung allerdings kaum tangieren. Es fehlt hier einerseits eine Darstellung und analytische Aufarbeitung der Institutionen, die für die politischen Prozesse in Afrika vielleicht nicht besonders wichtig sind, aber dennoch für die Erklärung der Herausbildung des autoritären Präsidentialismus sowie der überhöhten Rolle der Militärs eine wichtige Komponente bietet: die privaten Verbände der Unternehmer, Berufsgruppen und Bauern, die Gewerkschaften (die gelegentlich allerdings am Rande erwähnt werden) und Genossenschaften sowie der Justiz und der Massenmedien.

Wichtiger mag vielleicht noch ein anderer Einwand sein, dessen sich auch die Autoren bewußt zu sein scheinen (S. 4, 197). Die Herrschaftssynthese zwischen internationalen und nationalen Systemen wird von ihnen nur in einem knappen (vier Seiten langen) Schlußkapitel angesprochen. Sie ist in der übrigen Darstellung (von den historischen Kapiteln einmal abgesehen) also kaum präsent und erscheint somit nur als Randphänomen. Nuscheler/Ziemer suchen dies damit zu begründen, daß der Handlungsspielraum der regierenden Eliten gegenüber dem im Lande operierenden Auslandskapital und vor allem gegenüber den Mechanismen des Weltmarktes sehr begrenzt sei(S. 197/198). Bei ihnen heißt es: ,, Wenn in den voranstehenden Kapiteln die Machtkonzentration und Oligarchisierung des Entscheidungsprozesses im afrikanischen Präsidentialismus . . . als Kennzeichen afrikanischer Verfassungspolitik hervorgehoben wurde, dann wurde dieses Machtpotential am institutionellen Innenverhalten sowie am Verhältnis zwischen der Machtelite und der regierenden Masse festgemacht.“(S. 197) Damit wurde die Verknüpfung der verschiedenen Ebenen (Weltmarkt, nationale Eliten, Masse der Bevölkerung) und eben die Herrschaftssynthese zwischen nationaler und internationaler Ebene ausgeblendet.

So ist es denn nicht unproblematisch, die unterschiedlichen Handlungsspielräume des peripheren Staates und der politischen Machteliten nicht als zu prüfende Hypothese, sondern als Prämisse anzunehmen. Die Allokation von Werten und Zielen des Staates sowie auch deren Implementierung durch ausländische Berater, Soldaten, Entwicklungshilfeagenturen, IWF, Weltbank, ILO-Missionen usw. wird so gänzlich vernachlässigt, die von außen z. T. abgeleitete Legitimität of t brüchiger, ohne spezifischer Klassenbasis operierender Regime durch ausländische Interventionstruppen (übrigens nicht nur westlicher, sondern gelegentlich auch afrikanischer und zunehmend auch sozialistischer Herkunft), Militär- und Wirtschaftshilfe wird nicht thematisiert; die Verknüpfung von Weltmarktentwicklung und politischer Stabilität bzw. Destabilisierung dieser Länder (vermittelt etwa über einzelne erratische Rohstoffmärkte auf monokulturelle Exportländer) wird übersehen. Der Rezensent teilt die Skepsis der Autoren, hier einer deterministischen Agententheorie aufzusitzen (die „,Komparadorenbourgeoisie als Handlanger von . . ."). Dennoch sollten diese Zusammenhänge empirisch aufgearbeitet und theoretisch diskutiert werden.

Die ,Einleitung“ nimmt kaum $10 \%$ dieser beiden opulenten Bände ein. Der Schwerpunkt liegt in den Länderbeiträgen, auf die im Durchschnitt jeweils 40 Seiten - bei einer Varianz von 6 Seiten (Srao Thomé und Príncipe) bis 116 Seiten (Algerien) - entfallen. Diese sind damit so ausführlich gehalten, daß ein Leser mit allgemeinem Informationsstand über die politische Entwicklung Afrikas hier manche ,Entdeckungen“ machen kann und in jedem Fall ausführlicher informiert wird als in anderen Lexika und Sammelbänden. Andererseits sind 
sie aber doch knapper geschrieben und damit zeitsparender zu lesen als einschlägige Ländermonographien (die zudem über viele Staaten gar nicht vorliegen).

Alle Beiträge berichten chronologisch-historisch über die politische und verfassungsrechtliche Entwicklung meist seit Beginn der Kolonialzeit. Der Schwerpunkt der Darstellungen liegt im allgemeinen auf der jüngeren Entwicklung. Die Texte werden durch Karten und Graphiken sowie eine ausführliche Wahlstatistik aufgelockert und belegt und durch eine umfangreiche (Auswahl-)Bibliographie, in der Quellen zum Verfassungs- und Wahlrecht, amtliche Reporte und Quellenpublikationen, Sekundärliteratur zur Kolonial- und Politischen Geschichte, Wirtschafts- und Sozialstruktur, Parteiensystem und Wahlstudien aufgeführt werden, ergänzt.

Die Beiträge sind durchgehend kompetent geschrieben. In den Ländern, in denen der Rezensent sich einigermaßen auskennt, konnten kaum größere Schnitzer festgestellt werden; die übrigen boten durchgängig eine anregende und informative Lektüre. So sehr man den Herausgebern und Hauptautoren (wie natürlich auch den anderen hier mitarbeitenden Verfassern) zu diesem großen Wurf beglückwünschen kann, so sehr muß man sie (wie ihre potentiellen Leser) ob der unsinnigen verlegerischen Konzeption bedauern. Dieses Opus gehört in den Handapparat jedes Afrikawissenschaftlers und Praktikers. Der hohe Preis wird aber schon viele Institutsbibliotheken abschrecken, die beiden Bände anzuschaffen. Obwohl die Daten nicht ,,veralten“, geht die Entwicklung natürlich weiter. Gerade ein derartiges Handbuch, was seinen Wert durch die chronologisch-historische Darstellung der politischen Entwicklung erfährt, vermag die Bedürfnisse seiner Leser in dem Umfang nicht zu decken, wie es die neuere Entwicklung nicht mehr erfaßt. So enden z. B. die Beiträge über Ghana schon 1974, über Nigeria 1976. Was ist so z. B. in diesen beiden Ländern nicht alles passiert? Das Handbuch sollte daher durch fortlaufende Ergänzungen bzw. auch durch Neuauflagen von Zeit zu Zeit auf den neuesten Stand gebracht werden können. Dies scheint im vorliegenden Rahmen allerdings kaum möglich und auch nicht beabsichtigt zu sein. Dazu hätte es wohl der Auflage eines wohlfeileren Taschenbuches oder einer Loseblattsammlung oder dgl. bedurft. Schade, daß daran offenbar nicht gedacht worden war.

Rolf Hanisch

\section{A. B. Kasunmu (ed.)}

\section{The Supreme Court of Nigeria}

Heinemann Studies in Nigerian Law, Ibadan, 1977.

This most interesting book on the Supreme Court of Nigeria deals with a central probelm of modern African law, which can be described as the problem of survival of judicialism.

Another important contribution to this topic is B. O. Nwabueze's book entitled: "Judicialism in Commonwealth Africa. The Role of the Courts in Government." London 1977. The book on the Supreme Court of Nigeria has a foreword by Nabo B. GrahamDouglas of the Federal Ministry of Justice in Lagos. In this foreword the problem of judicial creation of law is envisioned. The book is edited by Kasunmu who also wrote the first contribution on the topic: "The Supreme Court of Nigeria: An Examination of its Composition and Functions." He shows interesting tables concerning the activities of the Court, for example Table B (p. 9) "Judges of the Court of Appeal (West)", or Table C (pp. 20) "Appeals to Supreme Court from High Courts and Courts of Appeal 1956-1970.” It is interesting to see that the number of cases disposed of between 1956-1970 did not change much: 1956 (120 cases), 1970 (130 cases). The highest number of cases disposed of occurred in 1958, followed by 1966 . The political development obviously diminished the number of disposed cases. Of 